\title{
Ultra-robust Wireless Interrogation for Sensors and Transducers: A Non-Hermitian Telemetry Technique
}

\author{
Minye Yang, Zhilu Ye, Mohamed Farhat, and Pai-Yen Chen*, Senior Member, IEEE
}

\begin{abstract}
We herein introduce a non-Hermitian parity-time $(P T)$-symmetric telemetry platform, capable of wirelessly and robustly acquiring information of passive sensors and chemiresistive or piezoresistive transducers. We theoretically and experimentally demonstrate that the $P T$-symmetric radiofrequency (RF) system, which consists of an active reader and a passive sensor to obey parity (space inversion) and time-reversal symmetries, may provide not only an improved sensitivity, but also rapid, accurate, and robust acquisition of sensor data. The unidirectional reflectionless properties arising from the spontaneous $P T$ symmetry-breaking may result in high-Q resonances and thus high resolvability in the measured reflection spectrum. Furthermore, the sensor's effective resistance (capacitance) can be wirelessly queried by electronically tuning the negative impedance converter (varactors) on the active reader, which allows observation of dual-band reflectionless phenomena. Unlike traditional telemetry systems, the measurement results are robust to variations in the inductive coupling strength, namely the distance and alignment between the two coil antennas. The proposed $P T$-symmetric telemetry paradigm may have an impact on a wide range of wireless sensing and instrumentation applications.
\end{abstract}

Index Terms - Wireless sensors, telemetry instruments, noninvasive measurement, near-field probing, radio-frequency sensors, parity-time symmetry, exceptional points

\section{INTRODUCTION}

5 $\mathrm{G}$ and beyond (5GB) wireless technologies provide wireless connectivity and continuous data gathering for a wide range of new applications in healthcare [1]-[4], vehicles [5], manufacturing industry [6], security [7], smart house automation [8], and internet-of-things (IoTs) [9]-[11]. In general, wireless sensing technology can be categorized into active and passive telemetry [12]. Active sensor telemetry systems could provide relatively long-range bidirectional sensor data transmission, which comes at the price of increased size and shortened lifetime due to their internal batteries (power source). Passive (battery-free) sensor telemetry systems, although providing reduced transmission distances, enable

\section{$\mathrm{M}_{\text {anuscript received at }}$}

M. Yang, Z. Ye and P. Y. Chen are with the Department of Electrical and Computer Engineering, University of Illinois at Chicago, Chicago, IL 60607, USA. (a)
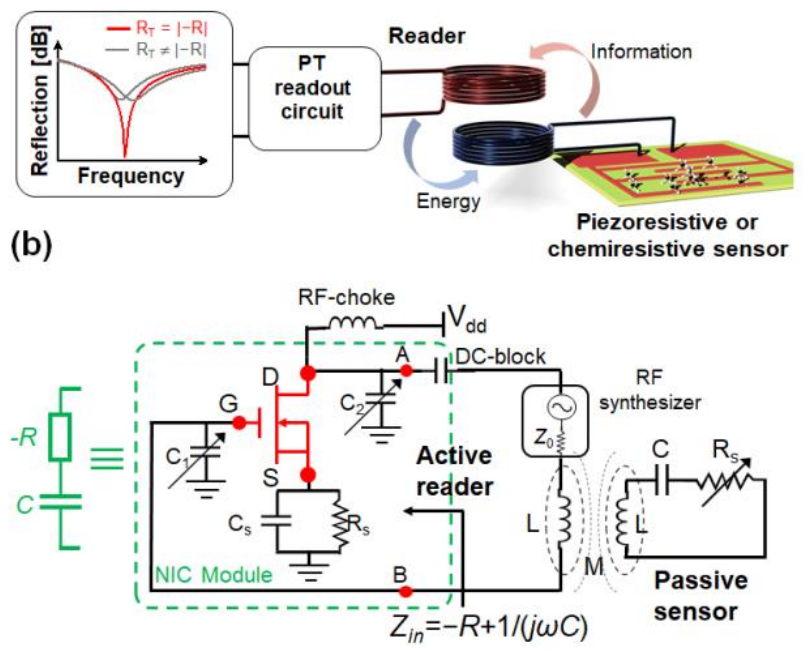

Fig. 1. (a) Schematics of proposed PT-symmetric wireless measurement system where an active reader is configured to wirelessly interrogate a passive sensor or transducer through inductive coupling. (b) Equivalent circuit diagram for the $P T$-symmetric telemetry system in (a).

indefinite lifetime and maintenance-free operation. Presently, there are two methods for implementing passive telemetry systems. One method is based on radio frequency identification (RFID) technology [10], [13]-[16], which monolithically integrates micromachined sensors with built-in signal conditioning units, analog-to-digital converters, and RF backscatter onto a chip to reflect a modulated RF signal toward the reader located in the far zone. Another fully passive sensor telemetry approach, which could be lightweight, low-cost, and minimally invasive, is on the basis of $R L C$ oscillator-based sensors that are embedded or implanted in the system to be monitored. These structures can be readily fabricated using micromachining and/or printed circuit technology. In principle, the physical parameter of interest could tune the resonant frequency of the $R L C$ sensor in the form of capacitive, resistive, or less commonly, inductive perturbation. Hence, the loci of the

M. Farhat is with the Computer, Electrical, and Mathematical Science and Engineering Division, King Abdullah University of Science and Technology (KAUST), Thuwal, 23955-6900, Saudi Arabia.

P.Y.C. would like to thank National Science Foundation Grant No. ECCSCCSS 1917678 for supporting this work.

Corresponding authors: P. Y. Chen; e-mail: pychen@uic.edu 
(a)

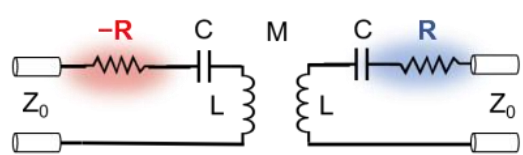

(b)

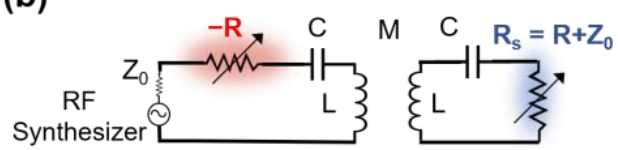

Fig. 2. (a) $P T$-symmetric electronic system realized with a two-port network. (b) Equivalent circuit model for the $P T$-symmetric wireless measurement system in Fig. 1; here, the active reader consisting of a coil antenna and a negative impedance converter is connected to a frequency synthesizer (e.g., vector network analyzer) with a generator impedance $Z_{0}$ to maintain $P T$ symmetry.

resonant frequency drift can be monitored in a contactless way by using a coil reader antenna inductively coupled to the $R L C$ sensor. Wireless passive $R L C$ sensors have been used for decades in monitoring of pressure [17], strain [18], food quality [19], temperature [20], environmental gases [21], bacteria [22], for which the environmental parameter to be measured is encoded in the reflection spectrum (i.e., search of peak resonant frequency). In the past few decades, $R L C$ oscillator-based wireless sensors and actuators have been extensively studied in terms of device's structural design, fabrication, and cost reduction. Despite tremendous research effort has already been done in how to achieve the robust wireless readout. In fact, size/geometrical constrains of the sensor and measurement setup usually give rise to low sensitivity and spectral resolvability because the constraint of quality factor is set by unavoidable skin-effect, Eddy current and/or dielectric losses. More critically, given that the resonant frequency shift has poor tolerance to misalignment between the sensor and reader coils in both vertical and axial directions, the absolute accuracy is sometimes questioned. A highly robust and accurate external readout circuit is thus an urgent demand for the practice of wireless $R L C$ sensors. In this paper, we will report a novel readout system for reliable and robust wireless query of passive $R L C$ sensors, such as micromachined capacitive transducers [23], [24] or piezo-/chemi-resistive sensors [25], [26]. The proposed telemetry system is inspired by parity-time (PT)symmetric non-Hermitian Hamiltonians in quantum mechanics, which can be readily implemented and tested in electronic and electromagnetic systems with loss and/or gain. Our approach enables continuous real-time wireless readout of sensors, with absolute accuracy that is independent of the alignment and interrogation range between two coils; namely, a wireless telemetry system that does not require sophisticated alignment, tilt-/range-control, and collimation of coils during the interrogation process.

Ever since $P T$-symmetric quantum systems were discovered by Bender and Boettcher in 1998, there have been abundant studies in the exotic physical phenomenon [27]-[30]: nonHermitian Hamiltonians may counterintuitively have entirely real eigenspectra once the system is invariant under combined operations of parity $(\mathcal{P})$ and time-reversal $(\mathcal{T})$ symmetry
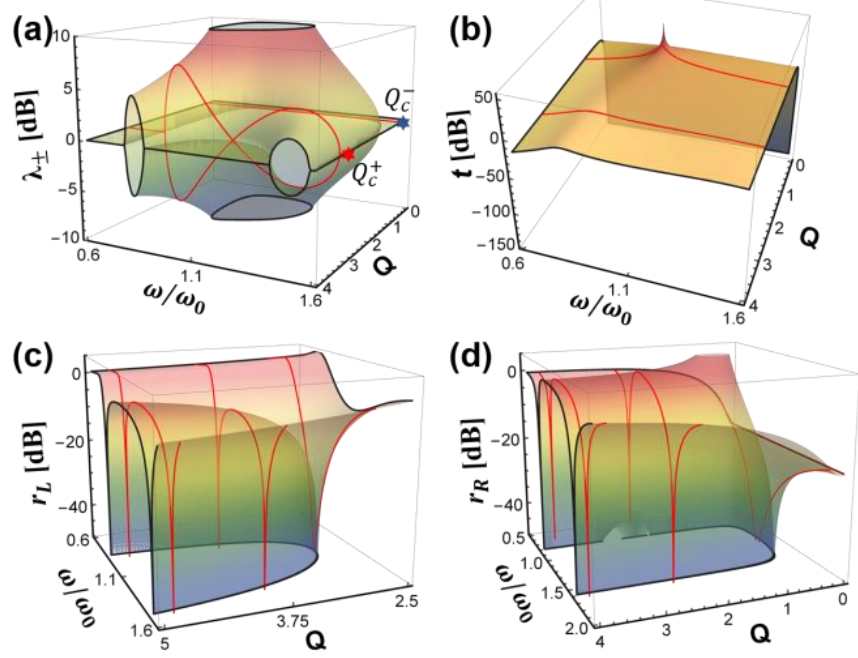

Fig. 3. (a) Contours of eigenvalues as functions of $Q$ and $\omega$ for the electronic $P T$ system in Fig. 2(a); here $\kappa=0.6$ and $\eta=1.2$. The critical $Q_{\mathrm{c}}^{ \pm}$are marked out by red and blue stars, respectively. (b)-(d) Corresponding transmission coefficient $(t)$, reflection coefficients at the left $\left(r_{L}\right)$ and right $\left(r_{R}\right.$ ) ports as functions of $Q$ and $\omega$ for the $P T$ system in (a).

[27], [28]; the former operator is a linear operator performing spatial inversion to transform coordinates and momenta, whereas the latter operator is an antilinear operator conducing the time inversion. Given that the antilinear character of $P T$, the Hamiltonian and the $P T$ operator do not always share the same eigenvectors. As a matter of fact, beyond certain nonHermiticity thresholds, abrupt phase transitions could occur, and the eigenvalues become complex. These spontaneous $P T$ symmetry-breaking points are referred to as exceptional points (EPs), and their existence in non-Hermitian systems may lead to an interesting phenomenon of having both eigenvalues and eigenvectors converge simultaneously [27]-[30]. Although the concept of $P T$-symmetric quantum systems is still a matter of debate, its electromagnetic[31]-[34], optical [35]-[38], acoustic [39], and electronic analogues [40]-[46] have been experimentally demonstrated. Electronic and electromagnetic measurement systems, although seemingly unrelated to the quantum theory, exhibit formal similarities between the Helmholtz and Schrödinger equations, essentially originating from the isomorphism of the two equations, i.e., Helmholtz equation governs vector fields of a monochromatic electromagnetic wave, and the time-independent Schrödinger equation describes the wavefunction of a massive particle. Further, electronic circuitry, which can be described by the telegraph equations (transmission line networks) and Kirchhoff's Laws (e.g., equivalence of wireless sensor telemetry and lumped-element circuitry) has emerged as a promising platform for observing $P T$-symmetry and nonHermitian physics because there are many experimentally feasible ways to create balanced, spatially modulated gain and loss in electronics [40]-[46].

In this context, we herein present theory and practical implementation for the $P T$-symmetric telemetric sensing system that enables flash and robust data acquisition. Figs. 1(a) and 1(b) illustrate the proposed $P T$-symmetric telemetry 

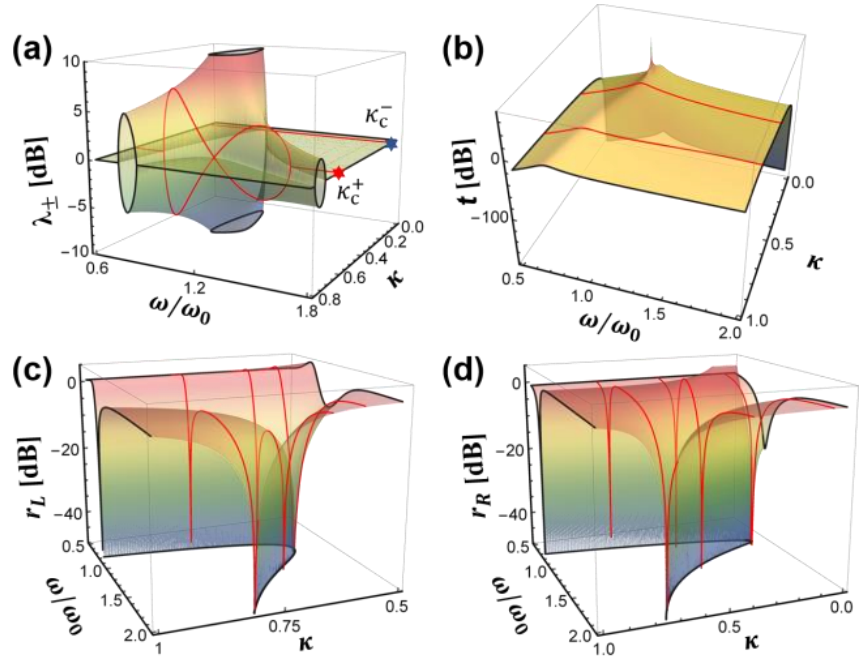

Fig. 4. (a) Contours of eigenvalues as functions of $\kappa$ and $\omega$ for the electronic $P T$ system in Fig. 2(a); here $Q=3$ and $\eta=1.2$. The critical $\kappa_{\mathrm{c}}^{ \pm}$are marked out by red and blue stars, respectively. (b)-(d) Corresponding transmission coefficient $(t)$, reflection coefficients from left $\left(r_{L}\right)$ and right $\left(r_{R}\right)$ as functions of $\kappa$ and $\omega$ for the $P T$ system in (a).

instrumentation and its practically realized circuit diagram for wireless interrogation of passive sensors and transducers, respectively. The circuit topologies in Figs. 2(a) and 2(b) can satisfy the $P T$-symmetry condition: the input impedances seen looking either side have the same reactance and oppositely signed resistance of the same value, which is necessary for the time-reversal symmetry. Such a paired amplifying and attenuation oscillators wirelessly linked via inductive coupling could support unidirectional reflectionless propagation for radio waves, when connected to a two-port network. The active resistance can be realized with the classical cross-coupled (XC) pair [47] with, for example, an input impedance of $-1 /\left(2 g_{m}\right)$ or a Colpitts-type feedback circuit [48]. For practical sensor telemetry applications, the active reader represents the $-R L C$ oscillator connected to the vector network analyzer (VNA) with a port impedance $Z_{0}=50 \mathrm{Ohm}$, while the passive sensor, typically modeled as an $R L C$ oscillator, is terminated by a load resistance of $Z_{0}$ such that the $P T$-symmetry condition is valid, as illustrated in Fig. 2(b). In conventional passive sensing systems, the sensor information is generally encoded in the reflected signal, which can be amplitude and/or phase modulated by the effective impedance of $R L C$ sensor. A straightforward way to read the sensor's impedance variations is to observe the shift in the resonance frequency. However, the resonance frequency is quite sensitive to the alignment between two coil antennas because it could be readily shifted or split due to changes in mutual inductance [49], [50]. On the other hand, in our approach, the sensor information is interpreted by the absolute value of $-R$ or $C$ in the reader circuit, which enables rapid and precise contactless readout, with excellent lateral and vertical coil misalignment tolerances. In the following, we will present the theoretical foundation of $P T$-symmetric telemetric sensing technique in section II, followed by experimental validation in section III.

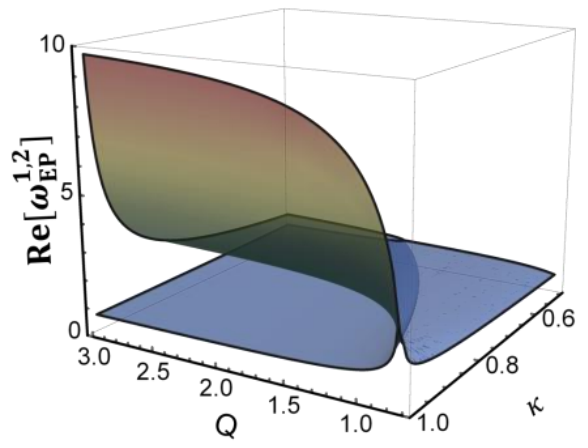

Fig. 5. Evolution of the normalized resonance frequencies (in unit of $\omega_{0}$ ) as a function of $Q$ and $\kappa$ for the $P T$ system in Fig. 2(a). There exist branch points at which the resonance frequencies shift drastically when $Q$ or $\kappa$ is varied.

\section{THEORY OF PT-SYMMETRIC TELEMETRY}

Based on the Kirchhoff's law [51], [52] and assuming a timeharmonic field of the form $e^{j \omega t}$, the scattering matrix $\mathbf{S}$ of the two-port $P T$ circuit sketched in Fig. 2(a) can be derived as:

$$
\begin{aligned}
& \mathbf{S}=\left[\begin{array}{cc}
t & r_{R} \\
r_{L} & t
\end{array}\right]=\frac{1}{a}\left[\begin{array}{cc}
j d & b-c_{-}^{2} \\
b-c_{+}^{2} & j d
\end{array}\right] ; \\
& a=b+j \frac{\omega^{2}-1}{\kappa \omega^{2}} d-c_{+} c_{-} ; \\
& b=Q^{2} \eta^{2}\left[\left(\kappa^{2}-1\right) \omega^{4}+2 \omega^{2}-1\right] ; \\
& c_{ \pm}=\omega(\eta \pm 1) ; \quad d=2 \eta Q \kappa \omega^{3},
\end{aligned}
$$

where $r_{L}$ and $r_{R}$ are the reflection coefficients for RF signal coming out of the left and right directions, respectively, and $t$ is the transmission coefficient (which is independent of the direction of arrival of incoming signals), the modified qualityfactor $Q=R^{-1} \sqrt{L / C}$ (which can be seen as the nonHermiticity of $P T$ system), the coupling strength $\kappa=M / L$ where $M$ is the mutual inductance between two coil antennas with a self-inductance $L, \eta=R / Z_{0}$, and $\omega$ is the angular frequency; here all frequencies are measured in unit of the natural frequency $\omega_{0}=1 / \sqrt{L C}$. Since the system is reciprocal, $t_{R}=t_{L}=t$, and the product of two reflections is given by: $\quad r_{R} r_{L}{ }^{*}=1-|t|^{2}$. In a typical passive network, flux conservation infers to the constraint $\mathbf{S}^{\dagger}(\omega) \mathbf{S}(\omega)=\mathbf{I}$, whereas in a $P T$-symmetric one, $\mathbf{S}$ in Eq. (1) satisfies: $\mathcal{P} \mathcal{T S}^{*}(\omega) \mathcal{P} \mathcal{T}=\mathbf{S}^{-1}(\omega)$ [36]. The transition between the exact $P T$-symmetric (exact $P T)$ phase and broken $P T$-symmetric $(P T$ broken) phase can be identified by the asymptotic behavior of eigenvalues of $\mathbf{S}$ [36], given by:

$$
\lambda_{ \pm}=t \pm \sqrt{r_{R} r_{L}}=\frac{j d \pm \sqrt{\left(b-c_{+}^{2}\right)\left(b-c_{-}^{2}\right)}}{2 a} .
$$

In the exact $P T$ phase, the two eigenvalues are nondegenerate and unimodular $\left(\lambda_{ \pm}=1\right)$, while the non-unimodular eigenvalues $\left(\lambda_{+}=1 /\left(\lambda_{-}\right)^{*}\right)$ suggest the $P T$-broken phase. At the EP, two eigenvalues are equal $\left(\lambda_{+}=\lambda_{-}=1 /\left(\lambda_{+}\right)^{*}\right)$; for 
(a)

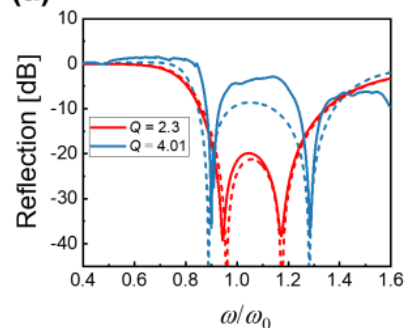

(b)

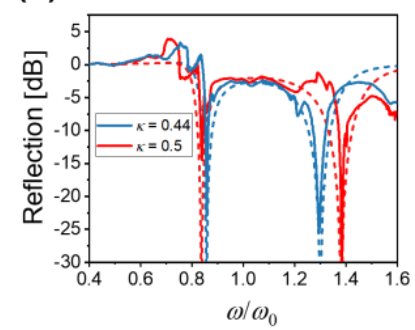

Fig. 6. (a) Reflection coefficients against frequency for different values of $Q$ and $\kappa=0.46$; here, the solid and dashed lines represent the experimental and theoretical results, respectively. (b) Similar to (a), but for different values of $\kappa$ and $Q=4.6$.

further details about $P T$ phase transition, see Appendix A. Fig. 3(a) presents evolution of eigenvalues as a function of $Q$ and the normalized frequency $\omega$; here, the coupling strength $\kappa=0.6$. As can be clearly seen from Fig. 3(a), the $P T$ phase transition is driven by the effective $Q$ of sensor and the frequency of operation. Figs. 3(b)-(d) present the corresponding transmission coefficient $(t)$ and the reflection coefficients $\left(r_{L}\right.$ and $r_{R}$ ) for the same system. It is seen from Figs. 3(a) and 3(b) that the change of the transmission coefficient from $|t|<1$ to $|t|>1$ is associated with the transition from the exact- $P T$ to $P T$-broken phase. Moreover, Figs. 3(b)-(d) clearly show that the reflectionless perfect transmission occurs in one direction but not the other, at the EPs where unimodular eigenvalues would transit to non-unimodular ones. The EP frequencies can be derived from Eq. (2) as:

$$
\omega_{E P}^{(1,2,3,4)}=\omega_{0} \sqrt{\frac{Q^{\prime 2}-1 \pm \sqrt{1-Q^{\prime 2}+4 Q^{\prime 4} \kappa^{4}}}{2 Q^{\prime 2}\left(1-\kappa^{2}\right)}},
$$

where $Q^{\prime}=(\eta / 1 \pm \eta) Q$ and the "+" and " -" signs of $Q^{\prime}$ in Eq. (3) correspond respectively to two different types of unidirectional reflectionless propagation: (1) $r_{L}=0$ and $r_{R} \neq 0 ;(2) r_{R}=0$ and $r_{L} \neq 0$; in both cases, $|t|=1$. The zero-reflection effect could be attributed to the step-functionlike behavior of the phase, which makes the delay time $\tau_{\text {delay }}=\partial \arg (r) / \partial \omega$ behave as a delta function and thus the reflected ac signals are trapped for a long time and being completely absorbed by the loss element [53]. It is also observed from Fig. 3(a) that there exists a threshold $Q$ value, beyond which real $\omega_{E P}$ can be found. The critical $Q$ values can be derived as:

$$
Q_{\mathrm{c}}^{ \pm}=\frac{1 \pm(1 / \eta)}{\kappa} \sqrt{\frac{1 \pm \sqrt{1-\kappa^{2}}}{2}} .
$$

If $Q \geq Q_{\mathrm{c}}^{+}$, EPs with the first kind of unidirectional reflectionless propagation $\left(r_{L}=0\right)$ can be observed; $Q_{c}^{+}$is marked as the red star in Fig. 3(a). In the same vein, if $Q \geq Q_{\mathrm{c}}^{-}$ , the second kind of unidirectional reflectionless transmission ( $r_{R}=0$ ) can also be obtained ( $Q_{\mathrm{c}}^{-}$is marked with the blue star in Fig. 3(a)). Fig. 4(a) presents evolution of eigenvalues of $\mathbf{S}$ as a function of the coupling strength $\kappa$ and frequency $\omega$; here $Q$ is fixed to 3. Those EPs observed in Fig. 4(a) can still be (a)

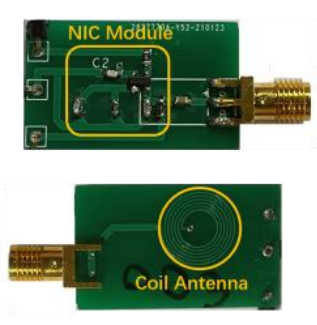

(b)

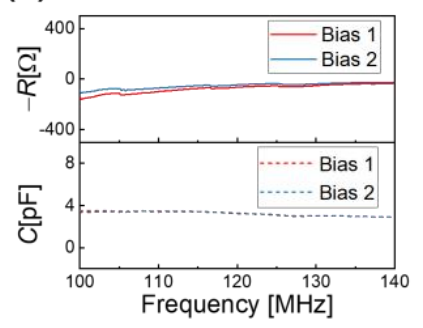

Fig. 7. (a) Photograph of the reader for $P T$-symmetric telemetric sensing system in Fig. 1. (b) Retrieved equivalent negative resistance (solid line) and equivalent capacitance (dashed line) of the negative impedance converter at different bias voltages.

described by Eq. (3). The critical coupling strength that enables the existence of real $\omega_{E P}$ can be derived as:

$$
\kappa_{\mathrm{c}}^{ \pm}=\frac{1 \pm(1 / \eta)}{Q} \sqrt{1-\left[\frac{1 \pm(1 / \eta)}{2 Q}\right]^{2}} .
$$

If $\kappa \geq \kappa_{\mathrm{c}}^{+}$, EPs with the first kind of unidirectional reflectionless property can be obtained. If $\kappa \geq \kappa_{\mathrm{c}}^{-}$, another EP pair that leads to the second kind of unidirectional reflectionless event is also obtained. Figs. 4(b)-4(d) report the transmission and reflection coefficients for the system in Fig. 2(a), showing that the EP frequencies associated with the existence of (unidirectional) reflection dips can be well described using Eqs. (3) and (5). We should note that for a specific $Q$ value, the magnetic coupling strength must be greater than $\kappa_{\mathrm{c}}$, such that EPs and sharp resonances (dips) can be obtained in the reflection spectra. Eq. (5) reveals that the interrogation distance between the reader and sensor coils must be sufficiently short if a low-Q oscillator is used. Fig. 5 shows the $\omega_{E P}^{(1,2)}$ as functions of $Q$ and $\kappa$. It is seen form Fig. 5 that there are branch points at which the $\omega_{E P}$ bifurcates and drastically varies with respect to changes in $Q$ and $\kappa$. Fig. 5 also presents the lower bound for $(Q, \kappa)$ which allows observation of EPs and the associated sharp dips in the reflection spectra. Finally, we note that for a very small ${ }_{\eta}, Q_{+}^{\prime} \approx Q_{-}^{\prime}$ and thus two different types of unidirectional reflectionless propagation could occur in the same frequency band. On the other hand, for a large ${ }_{\eta}$, resonant frequencies of two different reflectionless scenarios are far apart.

For practical wireless sensing applications, a single port setup is usually adopted, as shown in Fig. 2(b), and the sensor's information is encoded solely in the reflection spectrum. Preferably, passive sensing apparatus comprising a coil antenna and a sensor or transducer may represent the loss element in the $P T$-symmetric telemetry system. In this case, the equivalent circuit model should be slightly modified as follows: the reader connected to the signal generator (generator impedance $Z_{0}$ ) has an effective negative resistance of $-R$, whereas the equivalent resistance of the sensor or transducer $R_{s}=R+Z_{0}$. This results in the $P T$-symmetry condition illustrated in Fig. 2(b), namely $|-R|=R=R_{s}-Z_{0}$, and the reactive/inductive elements in the sensor and the reader are identical. By doing so, the reflection of the system is the same as $r_{L}$ obtained from the above two- 


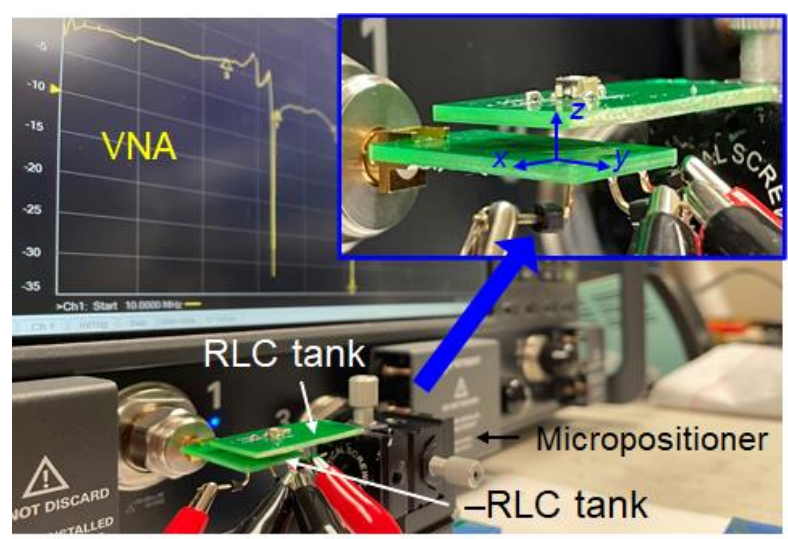

Fig. 8. Experimental setup of the $P T$-symmetric telemetric sensing system in Fig. 1, of which the active tank is directly connected to the VNA and the tunable passive tank as a pseudo-transducer is mounted on a micro positioner.

port analysis. When $Q$ is varied due to changes in the resistance (capacitance) of the sensor, the $-R(C)$ on the reader should be adjusted accordingly to maintain the $P T$-symmetry. Under a constant $\kappa$, the resistance variation $(\Delta R)$ or capacitance variation $(\Delta C)$ of the sensor can be characterized by tracking shifts of the two EP frequencies (i.e., loci of the two resonance frequencies), as shown in Fig. 6(a). This readout method, however, requisites a delicately aligning and pitching between two coil antennas because $\omega_{E P}$ is rather sensitive to $\kappa$, as illustrated in Fig. 6(b). This represents the major challenge for existing wireless passive $L C$ sensor systems. In the proposed $P T$-symmetric telemetry system, for a given $Q$ and an arbitrary $\kappa$, analysis of $\Delta R(\Delta C)$ of the sensor is done by reading the absolute $-R(C)$ value of the reader, instead of the $\kappa$ (distance)-dependent resonance frequency shift. Thanks to the symmetry between the absolute resistance and reactance of the reader and the sensor, the sensor's $\Delta R(\Delta C)$ can be contactlessly acquired by electronically sweeping $-R(C)$ of the reader until successful observation of two minima in the reflection spectrum (which means that two EPs are locked under the $P T$ symmetry condition). Since each $(Q, \kappa)$ set corresponds to a unique EP frequency pair, the relationship between the measured resonance frequencies and the values of $Q$ and $\kappa$ could be obtained with proper interpolation.

Consequently, our approach may offer a rapid and accurate wireless measurement, which is robust to misalignment between the coil antennas or variation in their separating distance, thus requiring no sophisticated alignments or positioning. On the other hand, conventional wireless sensing setups that rely on tracking the locus of impedance, phase, or peak frequency of a resonant mode do not provide similar absolute precision. There exists a general consensus that, to the best of the authors' knowledge, the data interpretation for traditional wireless passive sensor system is complicated by the fact that two different sets of $(Q, \kappa)$ could give the same resonance frequency. Additionally, traditional setups typically suffer from poor resolvability due to a rather broad spectral linewidth (low-Q resonance) [49], [54]. This, however, can be mitigated by the sharp reflectionless resonance in the electronic (a)

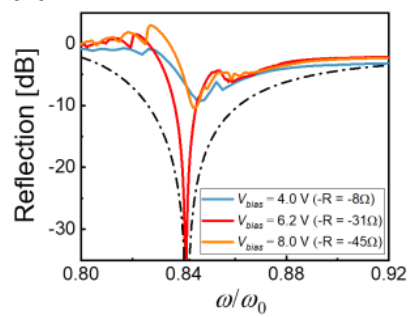

(b)

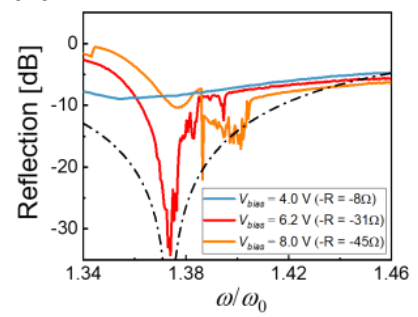

Fig. 9. (a) Reflection coefficients nearby the (a) first and (b) second resonance frequency under different bias conditions; here, the black dash-dot lines are the theoretical results obtained from $r_{L}$ Eq. (1). When the bias is tuned to 6.2 volts, at which $\left|-R-Z_{0}\right|=R_{S}$, the reflection dips are obtained, in consistent with the theoretical prediction in Eq. (3) and Fig. 5.

$P T$ system.

\section{EXPERIMENTS AND DISCUSSIONS}

We have conducted experiments for validating the proposed telemetric sensing method. In our laboratorial prototype, a reader and a pseudo-transducer were made using the printed circuit board (PCB) technology, as shown in Fig. 7(a). The selfinductance of the planar coil antenna and the mutual inductance between two coils can be determined by the Neumann formula [55]. In this work, the coil antenna self-inductance was designed to be $L=200 \mathrm{nH}$ and the magnetic coupling coefficient is tuned from 0 to 0.5 . We note that the coil antenna has a small parasitic resistance $R p \approx 0.2 \Omega$, retrieved from the scattering parameters. We have implemented a negative impedance converter (NIC) based on the Colpitts-type feedback oscillator shown in Fig. 1(b). In a Colpitts-type NIC, two variable shunt capacitors are connected parallel to the gate and drain terminals of a metal-oxide-semiconductor field-effect transistor (MOSFET; BF2040, fabricated by Infineon Inc.), as shown in Fig. 1(b) and Fig. 7(a). The input impedance seen into points A and B highlighted in Fig. 1(b) can be written as:

$$
\begin{aligned}
Z_{\text {in }} & =-R+\frac{1}{j \omega C} \\
& \approx-\frac{g_{m}\left(V_{\text {bias }}\right)}{\omega^{2} C_{1} C_{2}}+\frac{1}{j \omega\left[C_{1} C_{2} /\left(C_{1}+C_{2}\right)\right]} .
\end{aligned}
$$

In Eq. (6), we assume that the transconductance $g_{m} \gg \omega C_{g d}, \omega C_{g s}$ (i.e., the operating frequency far below the cutoff frequency), where $C_{g d}$ and $C_{g s}$ represent the gate-todrain capacitance and the gate-to-source capacitance, respectively. The NIC itself can be equivalent to a series combination of $-R$ and $C$ seen into the joint points [points A and B in Fig. 1(b)]. Fig. 7(a) shows the prototype of the NIC and the active reader in Fig. 1(b), which were implemented using the PCB technology. By connecting the gate and drain of the MOSFET to the VNA and the planer coil antenna, a $-R L C$ tank can be constructed. We note that a VNA (N5242B, Keysight Technologies, Inc) can be described by a negative resistance $-Z_{0}$ because it is regarded as an external source that steadily injects RF power to the system. Consequently, the net negative resistance of the active reader is $-R-Z_{0}$, which must be tuned to match the resistance of the passive sensor 


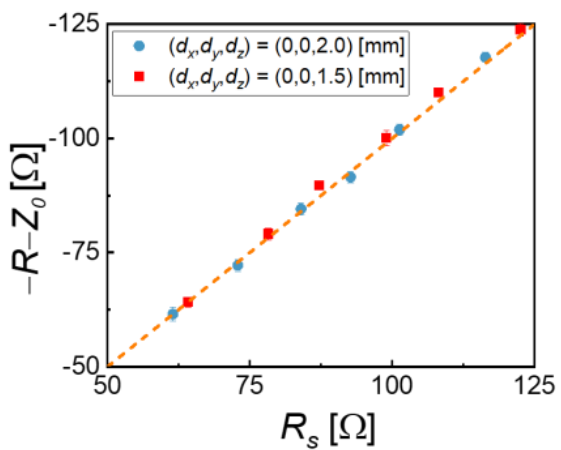

Fig. 10. Comparison between the measured and exact resistance of the pseudotransducer achieved by a variable resistor.

$R_{s}=R+Z_{0}$ such that gain-loss balance, necessary for $P T$ symmetry, can be achieved. The negative resistance and the input reactance of an isolated $-R L C$ tank can be measured directly using the VNA. Fig. 7(b) reports the measured input impedance of the Colpitts-type NIC at different DC biases that tune $-R$ of the NIC, showing that a stabilized and rather nondispersive series negative resistance (with a bandwidth of $\sim 40$ $\mathrm{MHz}$ ) and an input capacitance can be achieved. Here, we note that different DC bias conditions may not lead to noticeable changes in the equivalent capacitance of the NIC module, as described in Eq. (6).

Fig. 8 illustrates our telemetry experiment setup where the active $-R L C$ reader and the passive $R L C$ pseudo-transducer are inductively coupled to form the $P T$-symmetric electronic system in Fig. 1(a). Here, we used a variable resister in the $R L C$ tank for mimicking a chemiresistive or piezoresistive transducer (PVG3A101C01R00 fabricated by Bourns Inc.). Figs. 6(a) and 6(b) report the measured reflection coefficients for four different $(Q, \kappa)$ sets, showing a good agreement between the experimental and theoretical results and proving validity and effectiveness of our reader circuit. In our experiment, the alignment and distance between two coil antennas are randomized. Under certain coupling strength, the exact $Q$ and $\kappa$ values can be extracted by adjusting the bias conditions of the MOSFET till observation of two reflection dips on the VNA screen, associated with unidirectional reflectionless property at the $\omega_{E P}$ frequencies. Figs. 9(a) and 9(b) report variations of reflection coefficients around the two EP frequencies with different DC biases of the reader's NIC; here, $(Q, \kappa)=(4.7,0.49), R_{s}=81 \Omega, C=2.3 \mathrm{pF}$ and $L=330$ $\mathrm{nH}$. It can be seen from Fig. 9 that the first and second resonant dips are obtained when the bias is tuned to 6.2 volts, which is in consistent with what predicted by Eq. (3) (presented as the black dash-dot lines in Fig. 9). When the system is operating under the $P T$-symmetry condition, the two resonance frequencies ( $\omega_{1}$ and $\omega_{2}$ in Eq. (3)) can be correlated to the unique set of $(Q, \kappa)$. The effective resistance of the chemiresistor or piezoresistor is encoded in $Q \propto R^{-1}$.

Next, we intend to wirelessly read the resistance of the pseudo-transducer under an unknown coupling strength (distance) between reader and sensor coils, in order to valid the robustness and reliability of this alignment-free telemetry (a)

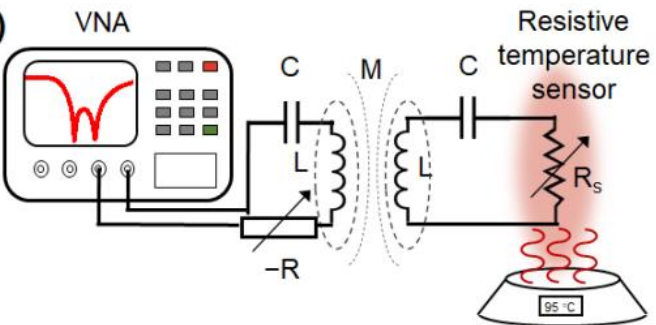

(b)
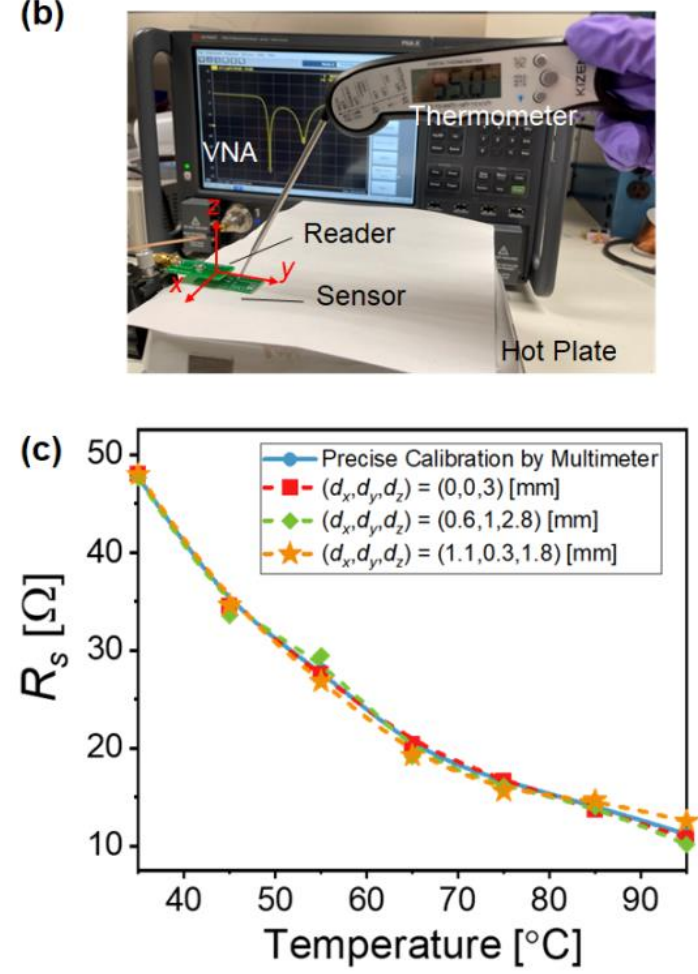

Fig. 11. (a) Schematic of the temperature sensing scenario using PT telemetry system and a negative temperature coefficient resistor. (b) The realistic experimental setup of (a). (c) The measurement data points by $P T$ telemetry system under different relative positions $\left(d_{x}, d_{y}, d_{z}\right)$ between the sensor of two coil antennas with fitting lines.

system. Here, the resistance of the pseudo-transducer is known by manually adjusting the variable resistor. Fig. 10 reports the measurement results for two random selected coil-to-coil distances, respectively represented by blue and red symbols. As mentioned above, under the condition of $P T$-symmetry, the sensor's effective resistance $R_{s}$ must be equal to the sum of equivalent negative resistance of NIC $-R$ and the negative resistance of the RF excitation source $-Z_{0}$ (e.g., $50 \Omega$ for VNA used here). From Fig. 10, it is evident that the measurement results show excellent linearity, implying that the sensor's resistance value can be read in a contactless way with high accuracy by simply citing the negative resistance value of NIC, i.e., $\left|-R-Z_{0}\right|=R_{s}$. Finally, we present a wireless temperature sensor composed of the same coil antenna and capacitor in Fig. 9 and a negative temperature coefficient resistor (NTC resistor: NCP15XC680E03RC, Murata Electronics Inc.). The schematics and photograph of the wireless temperature sensing setup are shown in Figs. 11(a) and 11(b), respectively. The resistance of the sensor drops from 
$50 \Omega$ to $10 \Omega$ when the substrate temperature increases from $35^{\circ} \mathrm{C}$ to $95^{\circ} \mathrm{C}$. In our experiment, heat is generated from a hot plate in the laboratory. The relationship between the resistance of NTC resistor and temperature is depicted by blue points in Fig. 11(c), which were obtained using the wired measurement with a digital multimeter. The red, green, and orange points in Fig. 11(c) are the results read wirelessly by the proposed $P T$ telemetry technqiue for different relative positions $\left(d_{x}, d_{y}, d_{z}\right)$ between the center of two coil antennas. Each data point in Fig. $11(\mathrm{c})$ is the average result of four measurements, with error bars represent the standard deviation of data. It can be evidently seen from Fig. 11(c) that accurate, repeatable, and reliable telemetry data acquisiton can be accomplished, regardless of whether the coils are well aligned. As a result, our telemetry technique provides a true alignment-free telemetric sensing, not possible with traditional wireless RLC sensor systems [49], [50]. Although in this work we demonstrated a resistive sensing scheme, the proposed telemetry method can also be applied to reactive sensing schemes where a capacitive sensor is commonly used to tune the system's $Q$ value (e.g., pressure and strain sensors [18], [56]). The accuracy can be further improved by optimizing the NIC design, e.g., implementation of NIC using complementary metal-oxide semiconductor (CMOS) technology that can minimize noises and fabrication errors, thereby better controlling the effective $-R$ and $C$ values. In addition to the precise control of resistive and reactive impedance values in the reader, the CMOS technology may also reduce the size and cost of readers and sensors, thus facilitating the practice of miniature wireless passive sensors in many applications. As representative examples, battery-free wireless $R L C$ microsensors have been widely used in monitoring the human organ pressures, such as intraocular pressure [50], intracranial pressure [57], blood pressure [58], and intestine pressure [59], to name a few. These kinds of sensors are also commonly used in industry and automotive applications, such as the tire pressure monitoring system. Last but not least, wireless passive $R L C$ sensors based on the Low Temperature Co-Fired Ceramics (LTCC) substrate can operate in harsh (e.g., high-temperature, toxic and hazardous) environments [60], where wire connection may not be possible. The proposed telemetry technique is foreseen to be beneficial for these fields.

\section{CONCLUSIONS}

We have proposed a robust, maker-free and alignment-free telemetric sensing technique, inspired by $P T$-symmetry discovered first in Schrodinger and Helmholtz systems. In the non-Hermitian $P T$-symmetric telemetric sensing system requiring a subtle gain-loss balance, the unidirectional reflectionless property can be found at the exceptional points, associated with a unique set of the coupling strength and the quality factor of electronic oscillators (i.e., a passive $R L C$ sensor and an active $-R L C$ reader, which are inductively or capacitively coupled). We demonstrate that at an arbitrary coilto-coil distance (i.e., magnetic coupling strength), changes in the effective impedance and $Q$-factor of an $R L C$ sensor can be accurately and wirelessly read by sweeping bias voltages of the reader circuit and recording the values that give resonant reflection dips related to exceptional points. Once exceptional points are captured, the absolute value of reader's negative resistance (capacitance) determined by the bias voltage of NIC (varactor) should be identical to the effective resistance (capacitance) of the sensor, which is the condition imposed by $P T$-symmetry. Such telemetry approach does not require tracing the resonance frequency drift or amplitude/phase of reflected signals at the resonant frequency, which are sensitive to both effective impedance of the sensor and the inductive coupling strength. Hence, the proposed telemetry technique may address the long-standing problem encountered by traditional wireless sensing systems, that is, the measurement results are very sensitive to the displacement and alignment of the reader antenna and, in general, the coil antennas must be precisely aligned and positioned. We envision that the proposed $P T$-symmetric telemetry technique may have an impact on the field of non-invasive and non-destructive wireless sensing for many real-world applications.

\section{APPENDIX: DetaILS ON PT PhaSE TRANSITION}

In the two-port scattering problems, $\mathbf{S}$ has eigenvalues in Eq. (2) and corresponding eigenvectors $\psi_{n}$, where $n \in\{1,2\}$. Let $\psi_{n}$ be an eigenvector of $\mathbf{S}$ corresponding to the eigenvalue $\lambda_{n}$ , we may obtain the following relationship:

$$
\begin{aligned}
& \mathbf{S} \mathcal{P} \mathcal{T} \psi_{n}=\mathcal{P} \mathcal{T}\left(\frac{1}{\lambda_{n}} \psi_{n}\right) ; \\
& \mathbf{S}\left(\mathcal{P} \mathcal{T}_{\psi_{n}}\right)=\frac{1}{\lambda_{n}^{*}} \mathcal{P} \mathcal{T} \psi_{n},
\end{aligned}
$$

where it is clear that $\mathcal{P} \mathcal{T} \psi_{n}$ is also an eigenvector of $\mathbf{S}$. Eq. (A1) is a weaker constraint than unitarity and can be satisfied in two possible ways: either each eigenvalue is itself unimodular, or the eigenvalues form pairs with reciprocal moduli, which correspond to exact- $P T$ and $P T$-broken phases [37]. First, in the exact $P T$ phase, $\mathcal{P} \mathcal{T}$ and $\mathbf{S}$ share the same eigenvectors, that is, ${ }_{\mathcal{P}} \mathcal{T}_{\mathcal{T}_{n}}= \pm \psi_{n}$. From (A1), we know that $\mathbf{S}\left( \pm \psi_{n}\right)= \pm \lambda_{n} \psi_{n}= \pm\left(\lambda_{n}^{*}\right)^{-1} \psi_{n}$, and therefore $\left|s_{n}\right|=1$. As a result, the spectrum of the $\mathbf{S}$ should be unimodular and nondegenerate. Physically, the eigenstate exhibits no net amplification nor dissipation in the $P T$-exact phase [36]. Second, in the $P T$-broken phase, $\psi_{n}$ is not $P T$-symmetric, but the eigenvectors satisfy $\mathcal{P} \mathcal{T}$ by transforming into each other: $\mathcal{P} \mathcal{T} \psi_{n}= \pm \psi_{m}$ and ${ }_{n} \neq m$. In such a phase, $\mathbf{S}\left( \pm \psi_{n}\right)= \pm\left(\lambda_{n}^{*}\right)^{-1} \psi_{n}$ and thus $\left|\lambda_{n} \| \lambda_{m}\right|=1$. The eigenvalues of the $\mathbf{S}$ are nonunimodular and a complex conjugate reciprocal pair. Physically, one mode of the system is localized in the gain side and thus exponentially amplified, while the other is localized in the loss side and thus dissipated. In other words, the eigenvectors of $\mathbf{S}$ cease to be $P T$-symmetric in the broken- $P T$ phase. Finally, the third case occurs when the eigenvalues and eigenvectors of $\mathbf{S}$ are degenerate, namely a transitional (exceptional) point which leads to spontaneously breaking of $P T$-symmetry. At the exceptional point, the two eigenvectors become degenerate and so do their eigenvalues. 


\section{REFERENCES}

[1] Y.-D. Lee and W.-Y. Chung, "Wireless sensor network based wearable smart shirt for ubiquitous health and activity monitoring," Sensors and Actuators B: Chemical, vol. 140, no. 2, pp. 390-395, Jul. 2009, doi: 10.1016/j.snb.2009.04.040.

[2] H. Huang, P.-Y. Chen, C.-H. Hung, R. Gharpurey, and D. Akinwande, "A zero power harmonic transponder sensor for ubiquitous wireless $\mu \mathrm{L}$ liquid-volume monitoring," Sci Rep, vol. 6, no. 1, p. 18795, May 2016, doi: 10.1038/srep18795.

[3] H. Huang et al., "RFID Tag Helix Antenna Sensors for Wireless Drug Dosage Monitoring," IEEE J. Transl. Eng. Health Med., vol. 2, pp. 18, 2014, doi: 10.1109/JTEHM.2014.2309335.

[4] Y. Xu et al., "Pencil-paper on-skin electronics," Proc Natl Acad Sci USA, vol. 117, no. 31, pp. 18292-18301, Aug. 2020, doi: 10.1073/pnas.2008422117.

[5] T. ElBatt, C. Saraydar, M. Ames, and T. Talty, "Potential for IntraVehicle Wireless Automotive Sensor Networks," in 2006 IEEE Sarnoff Symposium, Princeton, NJ, Mar. 2006, pp. 1-4. doi: 10.1109/SARNOF.2006.4534723.

[6] W. Li and S. Kara, "Methodology for Monitoring Manufacturing Environment by Using Wireless Sensor Networks (WSN) and the Internet of Things (IoT)," Procedia CIRP, vol. 61, pp. 323-328, 2017, doi: 10.1016/j.procir.2016.11.182.

[7] R. A. Potyrailo et al., "Wireless sensors and sensor networks for homeland security applications," TrAC Trends in Analytical Chemistry, vol. 40, pp. 133-145, Nov. 2012, doi: 10.1016/j.trac.2012.07.013.

[8] S. Pirbhulal et al., "A Novel Secure IoT-Based Smart Home Automation System Using a Wireless Sensor Network," Sensors, vol. 17, no. 12, p. 69, Dec. 2016, doi: 10.3390/s17010069.

[9] S. Li, L. D. Xu, and X. Wang, "Compressed Sensing Signal and Data Acquisition in Wireless Sensor Networks and Internet of Things," IEEE Trans. Ind. Inf., vol. 9, no. 4, pp. 2177-2186, Nov. 2013, doi: 10.1109/TII.2012.2189222.

[10] L. Zhu, H. Huang, M. M.-C. Cheng, and P.-Y. Chen, "Compact, Flexible Harmonic Transponder Sensor With Multiplexed Sensing Capabilities for Rapid, Contactless Microfluidic Diagnosis," IEEE Trans. Microwave Theory Techn., vol. 68, no. 11, pp. 4846-4854, Nov. 2020, doi: 10.1109/TMTT.2020.3006286.

[11] H. Huang et al., "Chemical-sensitive graphene modulator with a memory effect for internet-of-things applications," Microsyst Nanoeng, vol. 2, no. 1, p. 16018, Dec. 2016, doi: 10.1038/micronano.2016.18.

[12] X. Xu, J. Wan, W. Zhang, C. Tong, and C. Wu, "PMSW: a passive monitoring system in wireless sensor networks: PASSIVE MONITORING SYSTEM IN WIRELESS SENSOR NETWORKS," Int. J. Network Mgmt., vol. 21, no. 4, pp. 300-325, Jul. 2011, doi: 10.1002/nem.792.

[13] C. Cattuto, W. Van den Broeck, A. Barrat, V. Colizza, J.-F. Pinton, and A. Vespignani, "Dynamics of Person-to-Person Interactions from Distributed RFID Sensor Networks," PLoS ONE, vol. 5, no. 7, p. e11596, Jul. 2010, doi: 10.1371/journal.pone.0011596.

[14] L. Zhu, M. Farhat, Y.-C. Chen, K. N. Salama, and P.-Y. Chen, "A Compact, Passive Frequency-Hopping Harmonic Sensor Based on a Microfluidic Reconfigurable Dual-Band Antenna," IEEE Sensors J., vol. 20, no. 21, pp. 12495-12503, Nov. 2020, doi: 10.1109/JSEN.2020.3000778.

[15] L. Zhu, N. Alsaab, M. M.-C. Cheng, and P.-Y. Chen, "A Zero-Power Ubiquitous Wireless Liquid-Level Sensor Based on MicrofluidicIntegrated Microstrip Antenna," IEEE J. Radio Freq. Identif., vol. 4, no. 3, pp. 265-274, Sep. 2020, doi: 10.1109/JRFID.2020.3004351.

[16] K. Domdouzis, B. Kumar, and C. Anumba, "Radio-Frequency Identification (RFID) applications: A brief introduction," Advanced Engineering Informatics, vol. 21, no. 4, pp. 350-355, Oct. 2007, doi: 10.1016/j.aei.2006.09.001

[17] B. Nie et al., "Textile-Based Wireless Pressure Sensor Array for Human-Interactive Sensing," Adv. Funct. Mater, vol. 29, no. 22, p. 1808786, May 2019, doi: 10.1002/adfm.201808786.

[18] F. Stauffer et al., "Soft Electronic Strain Sensor with Chipless Wireless Readout: Toward Real-Time Monitoring of Bladder Volume," Adv. Mater. Technol., vol. 3, no. 6, p. 1800031, Jun. 2018, doi: 10.1002/admt.201800031.

[19] J. Koskela et al., "Monitoring the quality of raw poultry by detecting hydrogen sulfide with printed sensors," Sensors and Actuators B:
Chemical, vol. 218, pp. 89-96, Oct. 2015, doi: 10.1016/j.snb.2015.04.093.

[20] S. Bhadra, D. S. Y. Tan, D. J. Thomson, M. S. Freund, and G. E. Bridges, "A Wireless Passive Sensor for Temperature Compensated Remote pH Monitoring," IEEE Sensors J., vol. 13, no. 6, pp. 24282436, Jun. 2013, doi: 10.1109/JSEN.2013.2255519.

[21] Keat Ghee Ong, Kefeng Zeng, and C. A. Grimes, "A wireless, passive carbon nanotube-based gas sensor," IEEE Sensors J., vol. 2, no. 2, pp. 82-88, Apr. 2002, doi: 10.1109/JSEN.2002.1000247.

[22] K. G. Ong, J. Wang, R. S. Singh, L. G. Bachas, and C. A. Grimes, "Monitoring of bacteria growth using a wireless, remote query resonant-circuit sensor: application to environmental sensing," Biosensors and Bioelectronics, vol. 16, no. 4-5, pp. 305-312, Jun. 2001, doi: 10.1016/S0956-5663(01)00131-2

[23] B. T. Khuri-Yakub and Ö. Oralkan, "Capacitive micromachined ultrasonic transducers for medical imaging and therapy," J. Micromech. Microeng., vol. 21, no. 5, p. 054004, May 2011, doi: 10.1088/0960$1317 / 21 / 5 / 054004$.

[24] I. Ladabaum, Xuecheng Jin, H. T. Soh, A. Atalar, and B. t. KhuriYakub, "Surface micromachined capacitive ultrasonic transducers," IEEE Trans. Ultrason., Ferroelect., Freq. Contr., vol. 45, no. 3, pp. 678-690, May 1998, doi: 10.1109/58.677612.

[25] A. S. Fiorillo, C. D. Critello, and S. A. Pullano, "Theory, technology and applications of piezoresistive sensors: A review," Sensors and Actuators A: Physical, vol. 281, pp. 156-175, Oct. 2018, doi: 10.1016/j.sna.2018.07.006.

[26] S.-W. Chiu and K.-T. Tang, "Towards a Chemiresistive SensorIntegrated Electronic Nose: A Review," Sensors, vol. 13, no. 10, pp. 14214-14247, Oct. 2013, doi: 10.3390/s131014214.

[27] C. M. Bender and S. Boettcher, "Real Spectra in Non-Hermitian Hamiltonians Having P T Symmetry," Phys. Rev. Lett., vol. 80, no. 24, pp. 5243-5246, Jun. 1998, doi: 10.1103/PhysRevLett.80.5243.

[28] C. M. Bender, D. C. Brody, and H. F. Jones, "Complex Extension of Quantum Mechanics," Phys. Rev. Lett., vol. 89, no. 27, p. 270401, Dec. 2002, doi: 10.1103/PhysRevLett.89.270401.

[29] K. G. Makris, R. El-Ganainy, D. N. Christodoulides, and Z. H. Musslimani, "Beam Dynamics in P T Symmetric Optical Lattices," Phys. Rev. Lett., vol. 100, no. 10, p. 103904, Mar. 2008, doi: 10.1103/PhysRevLett.100.103904.

[30] R. El-Ganainy, K. G. Makris, D. N. Christodoulides, and Z. H. Musslimani, "Theory of coupled optical PT-symmetric structures," Opt. Lett., vol. 32, no. 17, p. 2632, Sep. 2007, doi: 10.1364/OL.32.002632.

[31] R. Fleury, D. L. Sounas, and A. Alù, "Negative Refraction and Planar Focusing Based on Parity-Time Symmetric Metasurfaces," Phys. Rev. Lett., vol. 113, no. 2, p. 023903, Jul. 2014, doi: 10.1103/PhysRevLett.113.023903.

[32] B. Peng et al., "Parity-time-symmetric whispering-gallery microcavities," Nature Phys, vol. 10, no. 5, pp. 394-398, May 2014, doi: 10.1038/nphys2927.

[33] Z. Ye, M. Yang, L. Zhu, and P.-Y. Chen, "PTX-Symmetric Metasurfaces for Sensing Applications," Frontiers of Optoelectronics, Feb. 2021, doi: 10.1007/s12200-021-1204-6.

[34] M. A. K. Othman and F. Capolino, "Theory of Exceptional Points of Degeneracy in Uniform Coupled Waveguides and Balance of Gain and Loss," IEEE Trans. Antennas Propagat., vol. 65, no. 10, pp. 52895302, Oct. 2017, doi: 10.1109/TAP.2017.2738063.

[35] C. E. Rüter, K. G. Makris, R. El-Ganainy, D. N. Christodoulides, M. Segev, and D. Kip, "Observation of parity-time symmetry in optics," Nature Phys, vol. 6, no. 3, pp. 192-195, Mar. 2010, doi: $10.1038 /$ nphys 1515.

[36] L. Ge, Y. D. Chong, and A. D. Stone, "Conservation relations and anisotropic transmission resonances in one-dimensional PT -symmetric photonic heterostructures," Phys. Rev. A, vol. 85, no. 2, p. 023802, Feb. 2012, doi: 10.1103/PhysRevA.85.023802.

[37] Y. D. Chong, L. Ge, and A. D. Stone, "P T -Symmetry Breaking and Laser-Absorber Modes in Optical Scattering Systems," Phys. Rev. Lett., vol. 106, no. 9, p. 093902, Mar. 2011, doi: 10.1103/PhysRevLett.106.093902.

[38] A. Regensburger, C. Bersch, M.-A. Miri, G. Onishchukov, D. N. Christodoulides, and U. Peschel, "Parity-time synthetic photonic lattices," Nature, vol. 488, no. 7410, pp. 167-171, Aug. 2012, doi: 10.1038/nature11298. 
[39] R. Fleury, D. Sounas, and A. Alù, “An invisible acoustic sensor based on parity-time symmetry," Nat Commun, vol. 6, no. 1, p. 5905, May 2015, doi: 10.1038/ncomms6905.

[40] M. Farhat, M. Yang, Z. Ye, and P.-Y. Chen, "PT-Symmetric AbsorberLaser Enables Electromagnetic Sensors with Unprecedented Sensitivity," ACS Photonics, vol. 7, no. 8, pp. 2080-2088, Aug. 2020, doi: 10.1021/acsphotonics.0c00514.

[41] M. Yang, Z. Ye, M. Farhat, and P.-Y. Chen, "Enhanced RadioFrequency Sensors Based on a Self-Dual Emitter-Absorber," Phys. Rev. Applied, vol. 15, no. 1, p. 014026, Jan. 2021, doi: 10.1103/PhysRevApplied.15.014026.

[42] Y. Ra'Di et al., "On-Site Wireless Power Generation," IEEE Trans. Antennas Propagat., vol. 66, no. 8, pp. 4260-4268, Aug. 2018, doi: 10.1109/TAP.2018.2835560.

[43] P.-Y. Chen et al., "Generalized parity-time symmetry condition for enhanced sensor telemetry," Nat Electron, vol. 1, no. 5, Art. no. 5, May 2018, doi: 10.1038/s41928-018-0072-6.

[44] M. Sakhdari, M. Hajizadegan, and P.-Y. Chen, "Robust extended-range wireless power transfer using a higher-order PT-symmetric platform," Phys. Rev. Research, vol. 2, no. 1, Art. no. 1, Feb. 2020, doi: 10.1103/PhysRevResearch.2.013152.

[45] Z. Ye, M. Yang, and P.-Y. Chen, "Multi-Band Parity-Time-Symmetric Wireless Power Transfer Systems," in 2021 IEEE Wireless Power Transfer Conference (WPTC), San Diego, CA, USA, Jun. 2021, pp. 14. doi: 10.1109/WPTC51349.2021.9457925.

[46] M. Sakhdari, M. Hajizadegan, Y. Li, M. M.-C. Cheng, J. C. H. Hung, and P.-Y. Chen, "Ultrasensitive, Parity-Time-Symmetric Wireless Reactive and Resistive Sensors," IEEE Sensors J., vol. 18, no. 23, Art. no. 23, Dec. 2018, doi: 10.1109/JSEN.2018.2870322.

[47] T. Soorapanth and S. S. Wong, "A 0-dB IL 2140 $\pm 30 \mathrm{MHz}$ bandpass filter utilizing Q-enhanced spiral inductors in standard CMOS," IEEE J. Solid-State Circuits, vol. 37, no. 5, pp. 579-586, May 2002, doi: 10.1109/4.997850.

[48] J.-A. Hou and Y.-H. Wang, "A 7.9 GHz Low-Power PMOS Colpitts VCO Using the Gate Inductive Feedback," IEEE Microw. Wireless Compon. Lett., vol. 20, no. 4, pp. 223-225, Apr. 2010, doi: 10.1109/LMWC.2010.2042559.

[49] R. Nopper, R. Niekrawietz, and L. Reindl, "Wireless Readout of Passive LC Sensors," IEEE Trans. Instrum. Meas., vol. 59, no. 9, pp. 2450-2457, Sep. 2010, doi: 10.1109/TIM.2009.2032966.

[50] P.-J. Chen, S. Saati, R. Varma, M. S. Humayun, and Y.-C. Tai, "Wireless Intraocular Pressure Sensing Using Microfabricated Minimally Invasive Flexible-Coiled LC Sensor Implant," $J$. Microelectromech. Syst., vol. 19, no. 4, pp. 721-734, Aug. 2010, doi: 10.1109/JMEMS.2010.2049825.

[51] S. Tretyakov, Analytical modeling in applied electromagnetics. Artech House, 2003.

[52] D. M. Pozar, Microwave engineering. Fourth edition. Hoboken, NJ : Wiley, [2012] C2012, 2012. [Online]. Available: https://search.library.wisc.edu/catalog/9910153599402121

[53] X. Zhu, H. Ramezani, C. Shi, J. Zhu, and X. Zhang, "P T -Symmetric Acoustics," Phys. Rev. X, vol. 4, no. 3, p. 031042, Sep. 2014, doi: 10.1103/PhysRevX.4.031042.

[54] B. George, H. Zangl, T. Bretterklieber, and G. Brasseur, "A Combined Inductive-Capacitive Proximity Sensor for Seat Occupancy Detection," IEEE Trans. Instrum. Meas., vol. 59, no. 5, pp. 1463-1470, May 2010, doi: 10.1109/TIM.2010.2040910.

[55] C. L. W. Sonntag, E. A. Lomonova, and J. L. Duarte, "Implementation of the Neumann formula for calculating the mutual inductance between planar PCB inductors," in 2008 18th International Conference on Electrical Machines, Vilamoura, Portugal, Sep. 2008, pp. 1-6. doi: 10.1109/ICELMACH.2008.4799978.

[56] Y. Peng, B. M. F. Rahman, T. Wang, G. Wang, X. Liu, and X. Wen, "Characterization of a Passive Telemetric System for ISM Band Pressure Sensors," J Electron Test, vol. 30, no. 6, pp. 665-671, Dec. 2014, doi: 10.1007/s10836-014-5485-1.

[57] N. H. Norager, A. Lilja-Cyron, T. S. Hansen, and M. Juhler, "Deciding on Appropriate Telemetric Intracranial Pressure Monitoring System," World Neurosurgery, vol. 126, pp. 564-569, Jun. 2019, doi: 10.1016/j.wneu.2019.03.077.

[58] B. Azizollah Ganji, S. Kheiry, and S. Soleimani, "Design of small size and high sensitive less-invasive wireless blood pressure sensor using MEMS technology," IET Circuits, Devices \&amp; Systems, vol. 13, no. 1, pp. 39-44, Jan. 2019, doi: 10.1049/iet-cds.2018.0013.
[59] P. Li, C. Kreikemeier-Bower, W. Xie, V. Kothari, and B. S. Terry, "Design of a Wireless Medical Capsule for Measuring the Contact Pressure Between a Capsule and the Small Intestine," Journal of Biomechanical Engineering, vol. 139, no. 5, p. 051003, May 2017, doi: 10.1115/1.4036260.

[60] J. Liu et al., "Fiber-optic Fabry-Perot pressure sensor based on lowtemperature co-fired ceramic technology for high-temperature applications," Appl. Opt., vol. 57, no. 15, p. 4211, May 2018, doi: 10.1364/AO.57.004211. 